\title{
Scopus- or SCIE-indexed journals
}

Miguel Abambres

The most "renowned" databases of scientific journals are Scopus and Science Citation Index Expanded (SCIE, popularly known as ISI), being the latter the most important for most academic/scientific institutions nowadays. 